\title{
Knowledge Regarding COVID-19 Pandemic among Student Nurses: Online Cross-sectional Survey
}

\author{
Abirami P. ${ }^{1}$, Suseelal T. ${ }^{2}$, Jelin Elizabeth G. ${ }^{3}$, Dr. C. Kanniammal ${ }^{3}$ \\ ${ }^{1}$ Professor, ${ }^{2}$ Asso. Prof., ${ }^{3}$ Asst. Prof, ${ }^{4}$ Dean, SRM College of Nursing, SRM College of Nursing
}

\begin{abstract}
COVID-19 pandemic it is a public health emergency declared by WHO and itis a major cause of concern for the healthcare profession. The present study is being conducted to assess the knowledge regarding COVID-19 Pandemic among student Nurses. A cross sectional survey by Using Online Google forms study was conducted. Non probability Convenient Sampling technique was used. The tool used for the study comprises of two sections. Section A comprises of demographic data and Section B comprises of structured questionnaire to assess the Knowledge regarding COVID-19 Pandemic among student Nurses which includes 25 questions. The data was collected and analysed by using descriptive and inferential statistics A structured questionnaire comprised of 25 questions developed by investigators was administered to 213 student Nurses. Among the 213 student Nurses, majority 56.8\% of them has adequate knowledge regarding the present global pandemic and $41.8 \%$ had moderately adequate knowledge. This study concludes that student Nurses adequate knowledge regarding COVID-19 pandemic.
\end{abstract}

Keywords: COVID-19 pandemic, Healthcare profession, Student Nurses.

\section{Introduction}

Coronaviruses are a large family of viruses that can cause illness ranging from the common cold to more severe diseases like Middle East Respiratory Syndrome (MERS) and Severe Acute Respiratory Syndrome (SARS). The 2019 novel coronavirus, called 'SARSCoV-2' (previously referred to as $2019-\mathrm{nCoV}$ ), is a new strain that has not been identified in humans before. The disease that is caused by SARS-CoV-2 is called 'COVID-19'.

On 31 December 2019, the World Health Organization (WHO) was informed of several cases of viral pneumonia of unknown cause detected in Wuhan City, China.

The outbreak has rapidly evolved, affecting other parts of China and many countries worldwide in Asia, Europe, North and South America, Australia and Africa. On 11 March 2020, WHO labelled the COVID-19 outbreak as a pandemic.

Typical symptoms of COVID-19 include fever, cough, difficulty breathing, muscle pain and tiredness.
More serious cases can develop severe pneumonia, acute respiratory distress syndrome, sepsis and septic shock. Anosmia-loss of the sense of smell or sometimes the sense of taste - has also been reported as a symptom of COVID-19 infection.

Generally, older people and those with underlying conditions (such as hypertension, heart disorders, diabetes, liver disorders, and respiratory disease) are expected to be more at risk of developing severe symptoms.

The evidence from analyses of cases to date is that COVID-19 infection causes mild disease (i.e. nonpneumonia or mild pneumonia) in about $80 \%$ of cases and most cases recover; $14 \%$ have more severe disease and $6 \%$ experience critical illness.

COVID-19 is transmitted from human-to-human mainly via flu-like symptoms and respiratory droplets that people sneeze, cough, or exhale. These droplets land on objects and surfaces, and others can catch COVID-19 by touching these objects or surfaces, then touching their eyes, nose or mouth. People can also catch COVID-19 
by breathing in droplets from a person with COVID-19 who coughs or exhales droplets.

The infectious period may begin 1-2 days before symptoms appear, but people are likely most infectious during the symptomatic period, even if symptoms are mild. The infectious period is estimated to last for 7-12 days in moderate cases and up to 2 weeks in severe cases $^{1}$.

The first case of COVID-19 in India, which originated from China, was reported on 30 January 2020. As of 15 June 2020, the Ministry of Health and Family Welfare (MoHFW) has confirmed a total of 332,424 cases, 169,798 recoveries (including 1 migration) and 9,520 deaths in the country ${ }^{2}$ India currently has the largest number of confirmed cases in Asia, ${ }^{3}$ and has the fourth highest number of confirmed cases in the world ${ }^{4}$ with the number of total confirmed cases breaching the 100,000 mark on 19 May and 200,000 on 3 June. ${ }^{5,6}$ India's case fatality rate is relatively lower at $2.80 \%$, against the global $6.13 \%$, as of 3 June. $^{7}$ Six cities account for around half of all reported cases in the country-Mumbai, Delhi, Ahmedabad, Chennai, Pune and Kolkata ${ }^{8}$. As of 24 May 2020, Lakshadweep is the only region which has not reported a case. On June 10, India's recoveries exceeded active cases for the first time reducing $49 \%$ of total infections. ${ }^{9}$

In response to this serious situation, the World Health Organization (WHO) declared it a public health emergency of international concern on January 30 and called for collaborative efforts of all countries to prevent the rapid spread of COVID-19

Maharashtra and Tamilnadu, has been seriously hit by the COVID-19 epidemic. Some unprecedented measures have been adopted to control the COVID-19 transmission all over India including the suspension of public transportation, the closing of public spaces, close management of communities, and isolation and care for infected people and suspected cases. Public were advised to just stay at home to avoid contacting with others.

The battle against COVID-19 is still continuing in India. To guarantee the final success, people's adherence to these control measures are essential, which is largely affected by their knowledge, towards COVID-19 in accordance with KAP theory ${ }^{10,11}$

As with SARS and MERS, when dealing with patients who may have COVID-19, health professionals should follow airborne precautions and wear respiratory masks (N95 or higher) during intubation and when entering a negative pressure room. As a Health Care professionals and the Future Nurses are responsible to update the knowledge on COVID 19 pandemic so this study aims to Investigate regarding Knowledge on COVID 19 pandemic among student Nurses at SRM college of Nursing.

\section{Materials and Method}

A quantitative approach was utilised to achieve the objectives of this study. Non experimental. Descriptive research design was adopted. Sample size was 213 Student Nurses. Based on the Non probability convenient sampling technique, inclusion and exclusion criteria samples were selected. The study variable was Knowledge on COVID-19 Pandemic .

Participants: This cross-sectional survey was conducted from March to April among Student Nurses the week immediately after the lockdown in India. Because it was not feasible to do a direct structured Interview method during this special period, we decided to collect the data online through Google forms from the Student Nurses as they are the Future Nurses. This Google form contained a brief Introduction on the background, objective, procedures, voluntary nature of participation, declarations of anonymity and confidentiality, and notes for filling in the questionnaire, and agreed to participate in the study were instructed to complete the questionnaire via clicking the link by the student Nurses. After confirmation of the question, the participant was directed to complete the self-report questionnaire. Totally 213 student Nurses responded to the Questionnaire.

Measures: The questionnaire consisted of two parts: demographics and Structured Questionnaire to assess the Knowledge on COVID-19 pandemic . Demographic variables included age, gender, marital status, level of education,Type of Family,Family History of Covid-19, Source of information regarding Covid-19,Dietary Pattern and place of current residence According to guidelines for the COVID-19 by the World Health Organisation, a knowledge questionnaire was developed by the authors. The questionnaire had 25 questions Overview, clinical presentations, transmission routes, prevention and control of COVID-19. These questions were created as Multiple Choice Question Method,a correct answer was assigned 1 point and an 
incorrect/unknown answer was assigned 0 points. The total knowledge score ranged from 0 to 25 . The Scoring Interpretation were 0 to 10 (Inadequate Knowledge), 11 to 20 (Moderately Adequate Knowledge),21 to 30 (adequate Knowledge) The Cronbach's alpha coefficient of the knowledge questionnaire was 0.75 in our sample, indicating acceptable internal consistency.

Statistical Analysis: The information collected from the study participants was scored and tabulated. The data were entered into the master coding sheet and saved in Microsoft Excel. Statistical analysis was conducted using Statistical Package for Social Sciences-16. Mean, percentage, and standard deviation were used to explain the demographic variables, and the Level of Knowledge on COVID 19 Pandemic among Student Nurses and Chi Square test to analyse the Knowledge on COVID 19 Pandemic among Student Nurses with their Demographic Variables.

\section{Results}

Table 1: Level of Knowledge regarding COVID-19

\begin{tabular}{|c|l|c|c|}
\hline S.No. & Level of knowledge & $\begin{array}{c}\text { No. of } \\
\text { respondents }\end{array}$ & Percentage \\
\hline 1 & Inadequate Knowledge & 3 & $1.4 \%$ \\
\hline 2 & $\begin{array}{l}\text { Moderately adequate } \\
\text { Knowledge }\end{array}$ & 89 & $41.8 \%$ \\
\hline 3 & Adequate Knowledge & 121 & $56.8 \%$ \\
\hline
\end{tabular}

\section{Discussion}

COVID-19 is a relatively new virus that has had devastating effects within the short time since it was first detected in December 2019. To date, there has been limited published data on knowledge regarding COVID 19 Pandemic. In this study predominantly we found an overall correct rate of $56.8 \%$ on the knowledge questionnaire, indicating that most respondents are knowledgeable about COVID-19. The finding of a high correct rate of COVID-19 knowledge among student nurses was unexpected, because this epidemiological survey was conducted during the very early stage of the epidemic. Because of the serious situation of the epidemic and the overwhelming news reports on this public health emergency, the student nurses would actively learn knowledge of this infectious disease from various channels of information. The $\mathrm{p}$ values corresponding to the demographic variable "Gender and Source of information regarding Covid-19" are less than 0.01 and are highly significant at $1 \%$ level hence we can say that there is high significant association between "Gender and knowledge level of COVID-19" and "Source of information regarding Covid-19 and Knowledge level of COVID-19". The significant positive association between social Media and COVID-19 knowledge scores supports this speculation

Similarly, the $\mathrm{p}$ value corresponding to the demographic variable "residential state" is less than 0.05 and is significant at $5 \%$ level hence we can say that there is significant association between "residential state and knowledge level of COVID-19".

All other $\mathrm{p}$ values are not significant at 5\% level since they are not less than 0.05 hence we can say that there is no significant association between "Age, Marital Status, Level of education, Types of family, Residence, Family History of Covid-19 and Dietary pattern" and "Knowledge level regarding COVID-19".

Thepresentstudy was ableto provide a comprehensive examination of the knowledge, toward COVID-19. The findings suggest that student Nurses have an acceptable level of knowledge on COVID-19 and are generally positive in their outlook on overcoming the pandemic. Even so, consistent messaging from the government and/or health authorities are key to aid their knowledge and understanding of COVID-19. Additionally, to update the Knowledge webinar on COVID 19 pandemic is conducted Continuously to improve the knowledge because the fact that Nurses are at risk of infection in the epidemic chain is a critical issue because Nurses help in controlling the outbreak.

Acknowledgement: The author acknowledges Dr. C. Kanniammal Dean, SRM College of Nursing for constant guidance, We would like to thank study participants for their constant support.

Conflict of Interest: Dr. Abirami P., Dr Suseelal T., Mrs. Jelin Elizebath G. and Dr. C. Kanniammal declares that no conflict of interest in addition, this study was not funded.

Statement of Human and Animal Rights: All procedures followed were in accordance with the ethical standards of the responsible committee on human experimentation (institutional and national) and with the Helsinki Declaration of 1975, as revised in 2008

Statement of Informed Consent: Informed consent was obtained from all the study participants for being included in the study. 


\section{References}

1. World Health Organisation, 9 ${ }^{\text {th }}$ March 2020, https://www.who.int/emergencies/diseases/novelcoronavirus-2019

2. "Home | Ministry of Health and Family Welfare | GOI”. mohfw.gov.in. Retrieved 15 June 2020.

3. "Number of Covid-19 cases in India climbs to 467, death toll rises to nine". livemint. 23 March 2020. Retrieved 26 March 2020.

4. "60-year-old Yemeni national dies due to coronavirus in Delhi”. Hindustan Times. 27 March 2020. Retrieved 30 March 2020.

5. "India most infected by Covid-19 among Asian countries, leaves Turkey behind". Hindustan Times. 29 May 2020. Retrieved 30 May 2020.

6. "India Crosses UK To Become Fourth Worst Hit By Coronavirus". NDTV.com. Retrieved 12 June 2020.

7. "India's case count crosses 100,000, Delhi eases restrictions: Covid-19 news today". Hindustan Times. 19 May 2020. Retrieved 20 May 2020.
8. Daily COVID-19 bulletin. "PIB India (@PIB India) on Twitter". Twitter. Retrieved 3 June 2020. [non-primary source needed]

9. "Infections over 1 lakh, five cities with half the cases: India's coronavirus story so far". The Week. Retrieved 20 May 2020.

10. Shivani Kumar, "Covid-19: Number of recoveries exceed active cases for first time". New Delhi: Hindustan Times. June 2020.

11. Ajilore K, Atakiti I, Onyenankey K. College students, "knowledge, attitudes and adherence to public service announcements on Ebola in Nigeria: Suggestions for improving future Ebola prevention education programmes. Health Education Journal. 2017;76:648-60

12. Tachfouti N, Slama K, Berraho M, Nejjari C, The impact of knowledge and attitudes on adherence to tuberculosis treatment: a case-control study in a Moroccan region. Pan Afr Med J. 2012;12:52 